\title{
Suppression of magnetic granularity by transport current in $(\mathrm{Bi}, \mathrm{Pb})_{2} \mathrm{Sr}_{2} \mathrm{Ca}_{2} \mathrm{Cu}_{3} \mathrm{O}_{x}$ tapes
}

\author{
A. E. Pashitski, A. Polyanskii, ${ }^{\text {a) }}$ A. Gurevich, ${ }^{\text {b) }}$ J. A. Parrell, and D. C. Larbalestier \\ Applied Superconductivity Center, University of Wisconsin, Madison, Wisconsin 53706
}

(Received 22 April 1995; accepted for publication 22 August 1995)

\begin{abstract}
Using the magneto-optical technique, transport current flow in $(\mathrm{Bi}, \mathrm{Pb})_{2} \mathrm{Sr}_{2} \mathrm{Ca}_{2} \mathrm{Cu}_{3} \mathrm{O}_{x}$ (BSCCO) $\mathrm{Ag}$-sheathed tapes has been imaged. For zero transport current, $I$, the magnetization currents display a pronounced macroscopic magnetic granularity (on a scale larger than the actual grain size) characterized by a percolative distribution of current loops. By applying $I \approx 0.8 I_{c}$ this granular behavior was largely replaced by a more uniform current flow. Due to the significant dependence of $I_{c}$ on $E$ in $\mathrm{BSCCO}$, the extent to which the magnetic granularity is observed strongly depends on the magnetic prehistory and on the electric field $E$, whose characteristic values are very different for magnetization and transport measurements. (C) 1995 American Institute of Physics.
\end{abstract}

The critical current density $J_{c}$ of Ag-sheathed $\left(\mathrm{Bi}_{1.8} \mathrm{~Pb}_{0.4} \mathrm{Sr}_{2} \mathrm{Ca}_{2} \mathrm{Cu}_{3} \mathrm{O}_{x}\right.$ (BSCCO-2223) tapes, is determined by both intrinsic and extrinsic factors. Intrinsic factors such as the short coherence length and high anisotropy result in strong flux creep and an essential dependence of $J_{c}$ on the electric field $E .^{1,2} J_{c}$ is also limited by the intergrain superconducting coupling both along the $c$-axis ${ }^{3}$ and in the $a b$ plane. ${ }^{4}$ Extrinsic factors, such as irregularities of the $\mathrm{Ag}-$ BSCCO interface, ${ }^{5}$ the grain-to-grain alignment, distributions of low- $T_{c}$ intergrowths ${ }^{6}$ and cracks ${ }^{7}$ play a significant role too. This results in strong inhomogeneities in $J_{c}(\mathbf{r})$, giving rise to percolative current flow ${ }^{7-10}$ and an effective current-carrying cross section smaller than the real cross section of the tape. Thus the identification of current paths in $\mathrm{BSCCO} / \mathrm{Ag}$ tapes is of great importance to understanding the real potential of high-temperature superconductors.

Recently, the magneto-optical (MO) technique has been employed to visualize the magnetic flux and current distributions in BSCCO tapes on $\sim 5 \mu \mathrm{m}$ scale. ${ }^{10,11}$ It was shown that $\mathbf{J}(\mathbf{r})$ could be deduced from the maps of $\mathbf{B}(\mathbf{r})$ and that the $J_{c}$ was markedly higher near the Ag-BSCCO interfaces, where colonies of well-aligned longer grains are mostly located. This observation verifies the inferences of previous microstructural $^{12,13}$ and electromagnetic ${ }^{7,14}$ studies. Central parts of the tapes having smaller grains and more second phase exhibited a pronounced magnetic granularity characterized by percolative current patterns consisting of an array of current loops much larger than the crystallites themselves. ${ }^{10}$

An important question is whether such pictures are equally valid in the presence of transport current (see also, Refs. 15 and 16). A fundamental difference between transport and magnetization measurements is that $E$ in transport measurements is generally much larger than in magnetization ones. Given the significant dependence of $J_{c}$ on $E$ in $\mathrm{BSCCO},{ }^{1,2}$ the transport current distribution $\mathbf{J}(\mathbf{r})$ might be very different. In this letter we show that the magnetic granularity in transport can be strongly suppressed, and that mag-

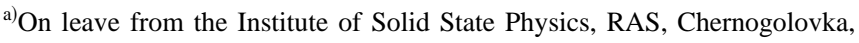
Moscow District, Russia.

${ }^{b)}$ Electronic mail: gurevich@ coefac.engr.wisc.edu
}

netization and transport behavior is indeed qualitatively different.

In this work, we used the MO technique to visualize magnetic flux penetration into a 2223 tape having a $50 \mu \mathrm{m}$ thick BSCCO core prepared by the "two-powder" process ${ }^{17}$ from the same batch studied in Ref. 10 for which $J_{c}(4.2 \mathrm{~K}, 0 \mathrm{~T})=90000 \mathrm{~A} / \mathrm{cm}^{2}$, and $J_{c}(77 \mathrm{~K}, 0 \mathrm{~T})=18000$ $\mathrm{A} / \mathrm{cm}^{2}$, at $E_{c}=1 \mu \mathrm{V} / \mathrm{cm}$. The sample was cut into a $10 \times 2$ $\times 0.2 \mathrm{~mm}$ slab. The long dimension $(10 \mathrm{~mm})$ was the tape axis $y$ along which the transport current $I$ was passed, the intermediate dimension $(2 \mathrm{~mm})$ was the $z$-axis along which the magnetic field $H_{a}$ was applied, and the shortest dimension $(0.2 \mathrm{~mm})$ was the $x$-axis, parallel to the $c$-axis of the grains. MO imaging of $B_{z}(\mathbf{r})$ from both magnetization and transport currents was performed by placing a $2 \mu \mathrm{m}$ thick Bi-doped $\mathrm{Y}_{3} \mathrm{Fe}_{5} \mathrm{O}_{12}$ film directly onto the polished central part of the $10 \times 0.2 \mathrm{~mm}$ surface of the sample. ${ }^{10,16} \mathrm{MO}$ images were taken for the magnetic field $H_{a}$ applied along the broad face of the tape parallel to the $a b$ planes and perpendicular to the indicator film (see Fig. 2)] at 14, 30, and 77 K, first in the zero-field cooled regime at $I=0$ and then after passing both dc and pulsed $I(t)$. The results were qualitatively similar for all temperatures studied, but we focus here on the $77 \mathrm{~K}$ data, which although more difficult to obtain due to the smaller $J_{c}$, are of greater technological interest.

Figure 1 shows MO images at $H_{a}=212$ Oe for $I=0$ and $I=10 A=0.8 I_{c}$, where darker regions correspond to lower local values of $B_{z}(\mathbf{r})$. It is striking that the highly nonuniform flux penetration at $I=0$ becomes much more uniform when a transport current is applied. Flux profiles taken at the sections marked in Fig. 1(b) are shown in Fig. 2. They indicate that the tape was well above the field of full flux penetration $(\approx 20-30$ Oe $)$, and that the self-field was $\approx H_{a} / 4$, the shape of $B_{z}(x)$ being in qualitative agreement with the Bean model (see inset). The irregularities of $d B_{z} / d x$ are due to inhomogeneities in $J_{c}(\mathbf{r})$. The mean slope $d B_{z} / d x$ in the region occupied by transport current was noticeably higher than in the region of reverse magnetization currents. We believe that this is a consequence of the higher $E$ of the transport measurement as compared to the more relaxed $E$ which correspond to magnetization currents. 


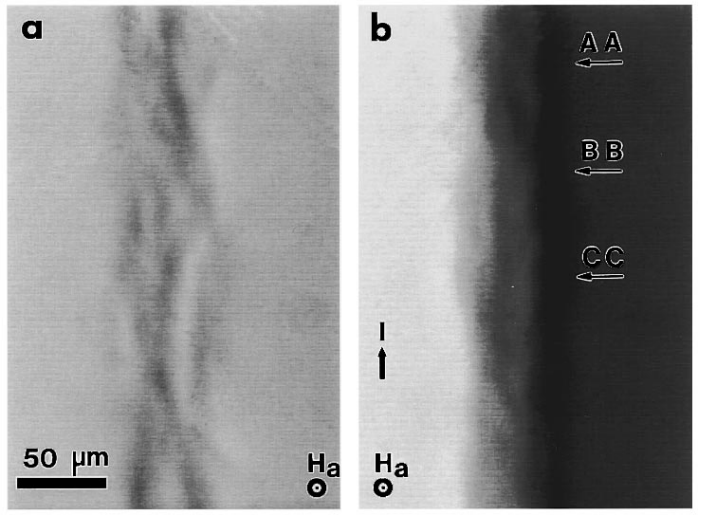

FIG. 1. MO images of the 2223 tape at $77 \mathrm{~K}$ and 212 Oe for transport current (a) $I=0$ and (b) $I=10 \mathrm{~A} \approx 0.8 I_{c}$.

To visualize the current flow, we plot the twodimensional (2D) map of $B_{z}(x, y)$ obtained from the MO images. The images were averaged over scales $L=6 \mu \mathrm{m}$, larger than the characteristic sizes of the second phase precipitates, as discussed in detail in Ref. 10. Due to the slab geometry of the sample and the high aspect ratio $(\sim 10)$ of the platelike grains whose long dimensions are parallel to $\mathbf{H}$, this averaging enables us to suppress the component $J_{z}(\mathbf{r})$, making $\mathbf{J}(\mathbf{r})$ effectively $2 \mathrm{D}$ over macroscopic scales $>L{ }^{10}$ Then the contour maps of $B_{z}(x, y)$ in Fig. 3 can be regarded as the current streamlines over lengths larger than $L$, although at the $\mathrm{Ag}-\mathrm{BSCCO}$ interface this interpretation is valid only qualitatively, since surface irregularities may cause noticeable $B_{x, y}(\mathbf{r})$ components. However, this $2 \mathrm{D}$ approximation certainly permits a clear qualitative visualization of current paths in both magnetization and transport experiments.

As seen from Fig. 3, the magnetic flux distribution at $I$ $=0$ is markedly different from that for the current-carrying state. At $I=0$, the tape exhibits a granular structure of magnetization currents characterized by an array of macroscopic current loops much bigger than the BSCCO grain size $(\approx 3$

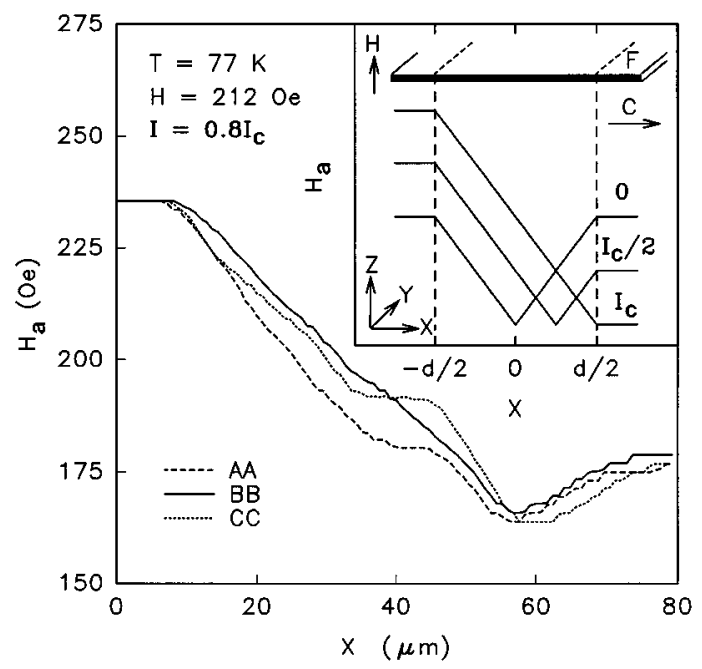

FIG. 2. Flux profiles taken at the marked places in Fig. 1(b). The inset shows $B_{z}(x, y, 0)$ for $I=0, I=I_{c} / 2$, and $I=I_{c}$ in the Bean model for a half-infinite slab in a parallel field. $F$ shows the location of the MO indicator film on the sample.

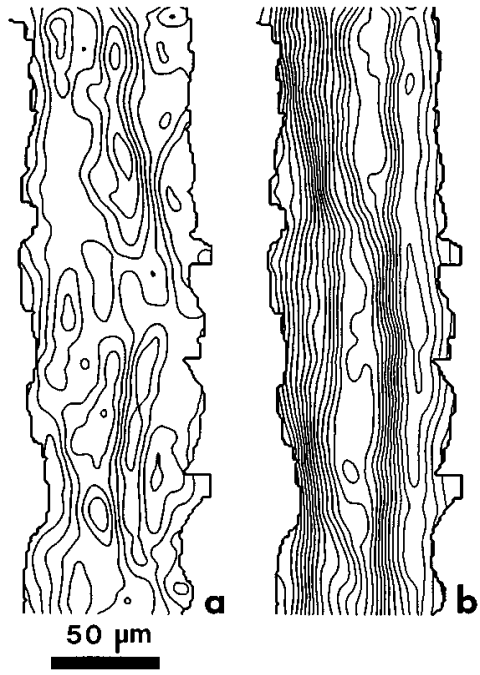

FIG. 3. Contours of constant $B_{z}(x, y)$ in steps of 3 Oe for (a) $I=0$ and (b) $I=0.8 I_{c}$ measured directly above the tape surface.

$\mu \mathrm{m})$. As was shown in Ref. 10, this magnetic granularity is determined by macroscopic inhomogeneities in $J_{c}(\mathbf{r})$ over a scale of several grains or grain colonies and depends on the magnetic prehistory. The transport current qualitatively changes this picture, eliminating most magnetization loops, except for some in the central part of the tape. This does indicate that the magnetic granularity on that scale is due to a metastable current structure which results from a nonuniform flux penetration during the magnetization process. Granular structure persists in the part of the tape which is not occupied by transport current and also in the central part which exhibits a rather granular behavior even at $I=0.8 I_{c}$. The latter is a feature of this particular tape which has a poor $J_{c}$ in the center due to the absence of colonies of wellaligned long grains and the presence of large second phase precipitates (or, perhaps, cracks) which give rise to significant deviation of the current streamlines. ${ }^{10}$

To evaluate the $J_{c}$ distribution, in magnetization and transport experiments, we plotted contour maps of the $J_{c}(x, y)$ surface calculated from the measured $B_{z}(\mathbf{r})$ in the 2D approximation, $J_{c}(\mathbf{r})=2\left[\left(\partial B_{z} / \partial x\right)^{2}\right.$ $\left.+\left(\partial B_{z} / \partial y\right)^{2}\right]^{1 / 2} \mu_{0}^{-1}$, where the factor 2 accounts for the volume occupied by currents which contribute to $B_{z}(x, y)$ on the surface of the semi-infinite slab in Fig. 2. The data shown in Fig. 4, indicate that, for $I=0$, the $J_{c}$ distribution consists of randomly scattered, disconnected high- $J_{c}$ regions which do not form a continuous percolative path along the tape. In this case the magnetic moment is mostly determined by the granular "white" regions which have $J_{c}<10^{4} \mathrm{~A} / \mathrm{cm}^{2}$. By contrast, in the current-carrying state shown in Fig. 4(b), the local $J_{c}$ values in the region occupied by transport current are considerably higher than for $I=0$, which appears to be due to the strong dependence of $J_{c}$ on $E$ at $77 \mathrm{~K}$ even for $H=212$ Oe. There is a clear boundary in Fig. 4(b) between the region of transport currents on the left-hand side, and the region of reverse magnetization currents on the right-hand side. Current patterns in Figs. 3 and 4 were imaged after significant flux creep occurred. This results in different magnitudes of $E(t)$ for transport and magnetization currents, since they correspond to very different initial values $E(0)$ 


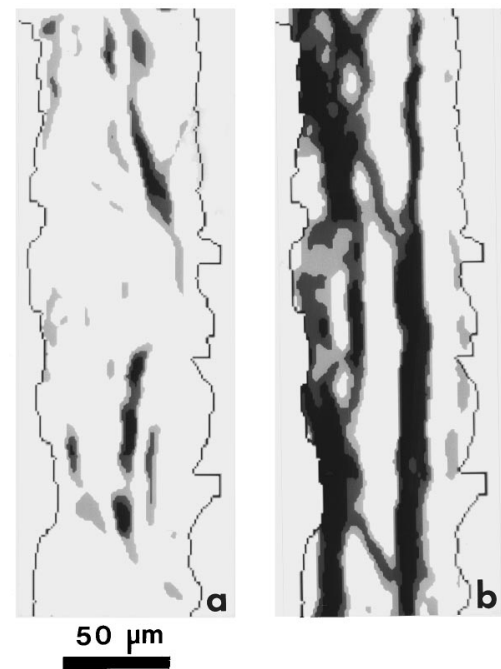

FIG. 4. 2D contour maps of the $J_{c}(x, y)$ surface for (a) $I=0$ and (b) $I$ $=0.8 I_{c}$. (b) Darker intensity corresponds to higher $J_{c}$ values, the white regions having $J_{c}<10^{4} \mathrm{~A} / \mathrm{cm}^{2}$. Three shades of gray correspond to $J_{c}$ values defined by the intervals $10^{4} \mathrm{~A} / \mathrm{cm}^{2}<J_{c}<2 \times 10^{4} \mathrm{~A} / \mathrm{cm}^{2}, 2$ $\times 10^{4} \mathrm{~A} / \mathrm{cm}^{2}<J_{c}<3 \times 10^{4} \mathrm{~A} / \mathrm{cm}^{2} ;$ and $J_{c}>3 \times 10^{4} \mathrm{~A} / \mathrm{cm}^{2}$ (black regions), respectively.

typical of magnetization and transport measurements. In addition, $I$ was applied several minutes after $H$ was applied, which also contributed to the more relaxed $E(t)$ for magnetization currents.

In conclusion, we have shown that a 2223 tape imaged under external field and zero transport current exhibits pronounced magnetic granularity with current loops having lengths of order the thickness of the BSCCO core. Transport current strongly suppresses this magnetic granularity, even at $77 \mathrm{~K}$. However, the $J_{c}$ values increase toward the $\mathrm{Ag}-$ BSCCO interfaces and the central part of the tape still has a low $J_{c}$. The data imply that BSCCO tapes having inhomogeneous $J_{c}(\mathbf{r})$ can exhibit qualitatively different transport and magnetization behavior. This fact may be very important for the correct interpretation of ac losses in BSCCO tapes, since the dissipation under ac magnetic field or ac transport current conditions may be different, as they correspond to very different structures of $\mathbf{J}(\mathbf{r})$ in Fig. 3

We are grateful to J. R. Clem and M. Polak for useful discussions and S. Dorris (ANL) for the supply of BSCCO powder. This work was supported by NSF MRG Program, DMR-9214707 (D.C.L., A.G.); ARPA, N00014-90 J-4115 (A.P.); and EPRI, RP8065-6 (A.E.P., J.P., D.C.L.).

${ }^{1}$ K. Shibutani, Q. Li, R. L. Sabatini, M. Suenaga, M. Motowidlo, and P. Haldar, Appl. Phys. Lett. 63, 3515 (1993); Y. Fukumoto, Q. Li, Y. L. Wang, M. Suenaga, and P. Haldar, Appl. Phys. Lett. 66, 1827 (1995).

${ }^{2}$ X. Y. Cai, A. Gurevich, D. C. Larbalestier, R. J. Kelley, M. Onellion, H. Berger, and G. Margaritondo, Phys. Rev. B 50, 16774 (1994).

${ }^{3}$ L. N. Bulaevskii, J. R. Clem, L. I. Glazman, and A. P. Malozemoff, Phys. Rev. B 45, 2545 (1992).

${ }^{4}$ B. Hensel, J. C. Grivel, A. Jeremie, A. Perin, A. Pollini, and R. Flükiger, Physica C 205, 329 (1993).

${ }^{5}$ K. Osamura, S. S. Oh, and S. Ochiai, Supercond. Sci. Technol. 5, 1 (1992).

${ }^{6}$ A. Umezawa, Y. Feng, H. S. Edelman, T. C. Willis, J. A. Parrell, D. C. Larbalestier, G. N. Riley, Jr., and W. L. Carter, Physica C 219, 378 (1994).

${ }^{7}$ D. C. Larbalestier, X. Y. Cai, Y. Feng, H. Edelman, A. Umezawa, G. N. Riley, Jr., and W. L. Carter, Physica C 221, 299 (1994).

${ }^{8}$ J. H. Cho, M. P. Maley, J. O. Willis, J. Y. Coulter, L. N. Bulaevskii, P. Halder, and L. R. Motovidlo, Appl. Phys. Lett. 64, 3030 (1994).

${ }^{9}$ A. Goyal, E. D. Specht, D. M. Kroeger, T. A. Mason, D. J. Dingley, G. N. Rilley, Jr., and M. W. Rupich, Appl. Phys. Lett. 66, 2903 (1995).

${ }^{10}$ A. E. Pashitski, A. Polyanskii, A. Gurevich, J. A. Parrell, and D. C. Larbalestier, Physica C 246, 133 (1995).

${ }^{11}$ U. Welp, D. O. Gunter, G. W. Crabtree, J. S. Luo, V. A. Maroni, W. L. Carter, V. K. Vlasko-Vlasov, and V. I. Nikitenko, Appl. Phys. Lett. 66, 1271 (1995).

${ }^{12}$ Y. E. High, Y. Feng, Y. S. Sung, E. E. Hellstrom, and D. C. Larbalestier, Physica C 220, 81 (1994).

${ }^{13}$ N. Merchant, J. S. Luo, V. A. Maroni, G. N. Riley, Jr., and W. L. Carter, Appl. Phys. Lett. 65, 1039 (1994).

${ }^{14}$ G. Grasso, B. Hensel, A. Jeremie, and R. Flükiger, Physica C 241, 45 (1995)

${ }^{15}$ V. K. Vlasko-Vlasov et al., Superconductivity 5, 1582 (1992); Th. Schuster et al., Phys. Status Solidi A 130, 429 (1992); M. V. Indenbom et al., J. Supercond. 6, 173 (1993).

${ }^{16}$ V. K. Vlasko-Vlasov, N. V. Goncharov, V. I. Nikitenko, A. A. Polyanskii, I. F. Voloshin, L. M. Fisher, N. M. Aleshina, and O. A. Poluschenko, Physica C 222, 367 (1994).

${ }^{17}$ S. E. Dorris, B. C. Prorok, M. T. Lanagan, S. Sinha, and R. B. Poeppel, Physica C 212, 66 (1993). 